\title{
CLINICAL APPLICATIONS OF NEXT-GENERATION SEQUENCING
}

\author{
Rosa Gloria Rebollar-Vega ${ }^{1}$, Cristian Arriaga-Canon² and Inti Alberto de la Rosa-VelázQuez ${ }^{1 *}$
}

${ }^{1}$ Genomics Laboratory, Red de Apoyo a la Investigación, Universidad Nacional Autónoma de México Instituto Nacional de Ciencias Médicas y Nutrición Salvador Zubirán, Mexico City; ${ }^{2}$ CONACYTInstituto Nacional de Cancerología, Mexico City, Mexico

\begin{abstract}
The significant drop in sequencing costs boosted by chemistries optimization and sample multiplexation has resulted in an immense growth within the field of next-generation sequencing (NGS) in the last decade. This has allowed a diversification of techniques, promoting a rapid advance in knowledge on the molecular basis of human disease. Due to the applicability and importance of this technology in basic research, it has quickly migrated to the clinical setting. NGS enables clinicians to make improved diagnostic and treatment decisions, which ultimately may influence precision medicine. (REV INVES CLIN. 2018;70:153-7)
\end{abstract}

Key words: Next-generation sequencing. Clinical diagnostics. Clinical genomics.

\section{INTRODUCTION}

Next-generation sequencing (NGS) technologies are being widely adopted in the clinical setting providing clinicians with improved tools to understand the complex mechanisms underlying patient's disease and facilitating further advances in early diagnostics, prediction of disease risk, and therapeutic decision making in a clear path toward precision medicine ${ }^{1}$.

The main applications of NGS in clinical diagnostics include the detection of mutations, splice variants, structural alterations, gene fusions, single nucleotide

\section{Corresponding author:}

*Inti Alberto de la Rosa-Velázquez

Red de Apoyo a la Investigación (RAI)

Instituto Nacional de Ciencias Médicas

y Nutrición Salvador Zubirán

Avenida Vasco de Quiroga, No.15

Col. Belisario Domínguez Sección XVI, Del. Tlalpan

C.P.14080, Mexico City, Mexico

E-mail: inti.delarosav@incmnsz.mx variations, small INDELs, copy number variations, comparison of expression profiles, and genome-wide association studies. The most used techniques include, but are not restricted to, whole-genome sequencing (WGS), whole-exome sequencing (WES), transcriptome sequencing, and a variety of targeted sequencing panels designed to analyze specific sets of genes, allowing greater sequencing depth and coverage and increased analytical sensitivity and specificity at a reduced cost (Table 1$)^{2}$. This technology has been widely adopted in a number of clinical and research fields including oncology, prenatal diagnosis, and microbiology among others, particularly because

Received for publication: 15-03-2018 Accepted for publication: 25-05-2018 doi: $10.24875 / R I C .18002544$ 
most of the NGS protocols can be applied with the established sample collection techniques from blood, buccal swab, saliva, skin punch, and tumors biopsies or formalin-fixed, paraffin-embedded tissue (Fig. 1).

\section{CLINICAL APPLICATIONS OF NGS}

The oncology field has benefited the most from NGS. The number of genomic alterations found by WGS and WES profiling from a variety of tumor samples and their corresponding normal tissues have scaled up in recent years. Furthermore, the use of NGS for hereditary cancer risk assessment is becoming a reality in cancer genetics, such that different leading cancer centers members of the National Comprehensive Cancer Network (NCCN) now include the use of multigene testing for familial breast and ovarian cancer risk assessment ${ }^{3}$. Recent technical advances allow the clinical scientist to perform genomic profiling on samples derived from small amounts of paraffin-embedded tumor tissue, single cells, or tumor-derived cellfree DNA from plasma with high sensitivity and specificity ${ }^{4}$. NGS can also help to elucidate the mechanisms of drug resistance and guide subsequent treatment, by identifying, for example, new mutations in biopsies at disease relapse, but the power of NGS in the clinical setting seems to focus on the gene panels, where oncologists may obtain data from a selected list of genes relevant for the specific type of tumor allowing a deeper coverage at reduced cost ${ }^{5}$. A variety of panels are available for different tumors including breast, colorectal, and familial cancer, among many others, and the U.S. Food and Drug Administration (FDA) has already approved some of these detection panels (Table 1 ). In this context, the use of NGS in the clinical setting represents a unique opportunity to explore the relationship between the defined molecular abnormalities and response to drugs with targeted mechanisms of action ${ }^{6}$. Considering these challenges, a number of multicenter Umbrella and Basket trials have been initiated. In the Umbrella trials, patients with a single tumor type are enrolled and treated depending on the different molecular biomarkers. On the other hand, in the Basket trials, patients with different tumor types are selected based on the same biomarker profile and receive a single treatment. The clinical trial $\mathrm{NCl}-\mathrm{MATCH}$ is a precision medicine cancer treatment where patients are assigned to receive therapy based on the genetic changes found in their tumors through genomic sequencing and other tests 6 . It is important to mention that these programs have implemented the integration of "Genomic/Tumor Boards", where the attending clinician and a panel of experts including oncologists, geneticists, bioinformaticians, and other experts discuss the molecular findings and make clinical recommendations to each patient.

In other areas, whole-genome and exome sequencing of fetal material derived from amniocytes or chorionic villi are being already performed in specialized centers, though it is not a routine practice. In this area, several factors must be considered including gestational age, sample type, and maternal cells, which may lead to DNA contamination. Nevertheless, prenatal genomic sequencing offers an opportunity for early intervention and a potentially higher likelihood of effective treatment ${ }^{7}$. Furthermore, precisely due to the greater sensitivity of NGS and the realization that fetal DNA is circulating in the mother's serum, we now see the emergence of the field of noninvasive prenatal testing as a more convenient and safe alternative to this invasive tests ${ }^{8}$.

On the other hand, NGS is also being used in medical microbiology for molecular diagnostics, outbreak monitoring, characterization and surveillance of pathogens, as well as detection of novel resistance genes. The most popular application is metagenomics, in which, for example, sequencing of the $16 \mathrm{~S}$ ribosomal subunit of bacteria may help in the identification of a diversity of species in different samples ${ }^{9}$. In addition, sequencing whole bacterial DNA or RNA from clinical samples by shotgun metagenomics may allow detecting the presence of pathogen-associated virulence and resistance genes.

In recent years, many groups have used NGS (WGS or WES) to identify disease-associated variants in a growing number of conditions, including neurological diseases such as spinocerebellar ataxia, Charcot-Marie-Tooth disease, hereditary spastic paraplegia, and amyotrophic lateral sclerosis, among others, providing an opportunity to discover new therapeutic strategies for these severe diseases. Another particular NGS application is the human leukocyte antigen (HLA) typing, which yields a comprehensive profiling of the HLA loci for applications in the field of organ transplantation. In fact, it has been recently adopted by the 
Figure 1. Applications of next-generation sequencing (NGS) in the clinical setting. (A) Applications available for the clinicians depend on the initial nucleic acid type, DNA (genomics), or RNA (transcriptomics). Samples can be obtained from different sources and tissues applying the already established protocols and analyzed in a global (whole-genome sequencing or wholeexome sequencing) manner or using specific gene panels. (B) The NGS workflow starts with the sample selection, purification, and quantification of the chosen nucleic acid and the selection of the appropriate library method, followed by sequencing and data analysis. Integration of a genomic board is crucial to review all the data and provides clinical recommendations that are delivered by the attending clinician.

A

Genomics

- WGS and WES

Point mutation

Indels

CNVs

Structural variations

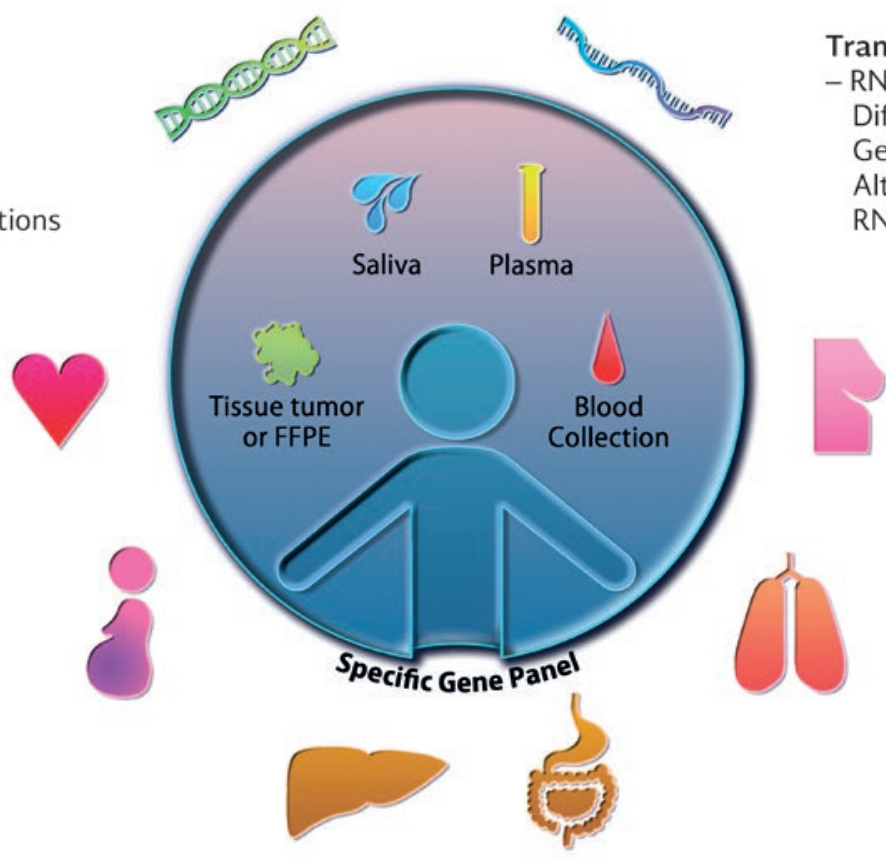

B

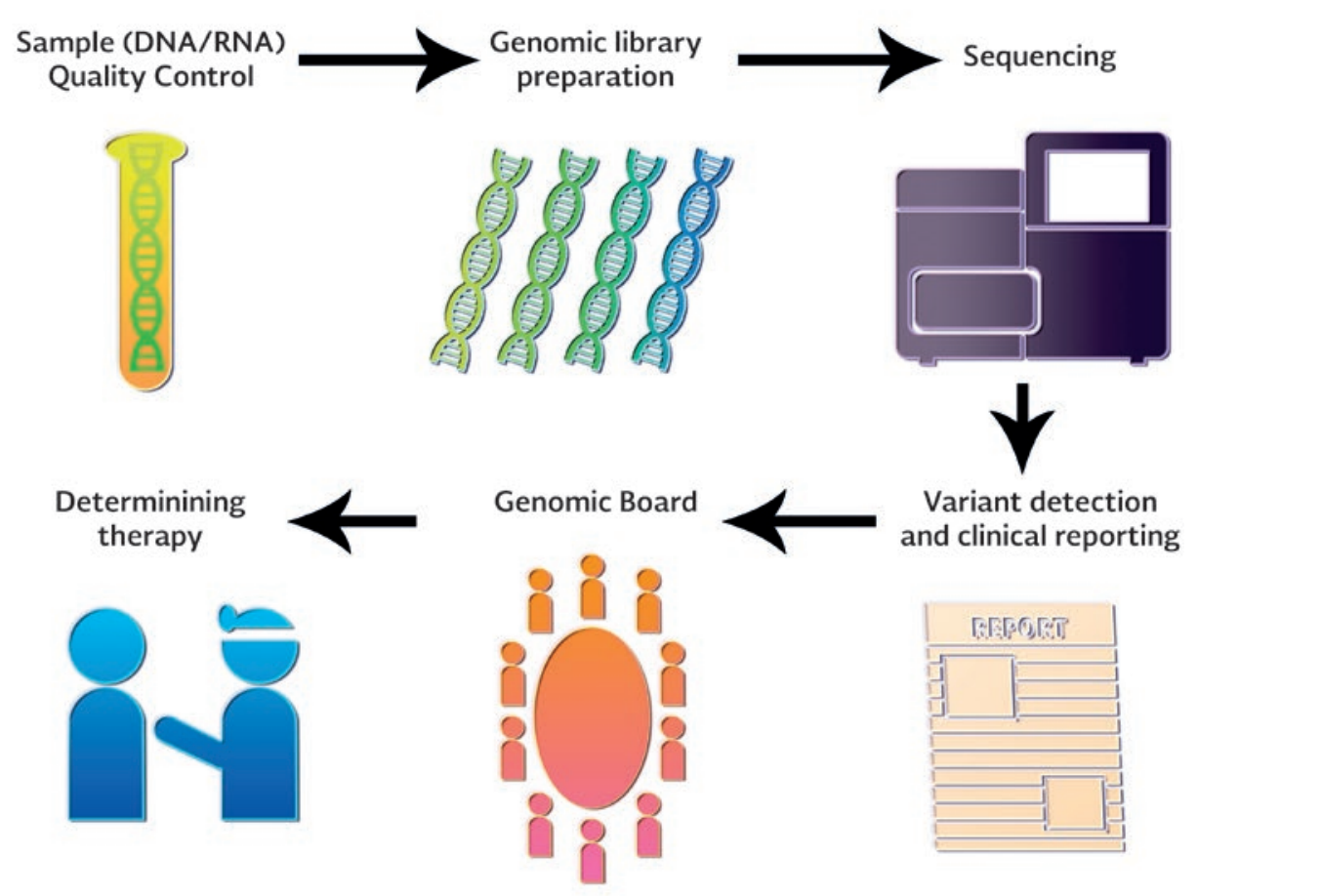


Table 1. Brief list of next-generation sequencing panels for clinical use.

\begin{tabular}{|c|c|c|c|c|c|}
\hline \multirow[t]{2}{*}{ Company } & \multirow{2}{*}{$\begin{array}{l}\text { Product } \\
\text { Cancer }\end{array}$} & \multirow[t]{2}{*}{$\begin{array}{l}\text { Number of } \\
\text { analyzed genes }\end{array}$} & \multirow[t]{2}{*}{ Target } & \multicolumn{2}{|c|}{$\begin{array}{l}\text { FDA } \\
\text { approved }\end{array}$} \\
\hline & & & & $\overline{\tilde{\sigma}}$ & \\
\hline Thermo Fisher & Oncomine $\mathrm{Dx}^{\mathrm{TM}}$ & 52 & $\begin{array}{l}\text { Hotspots, SNVs, INDELs, CNVs, } \\
\text { and gene fusions }\end{array}$ & $\frac{2}{(0)}$ & Yes \\
\hline Illumina & TruSight ${ }^{\oplus}$ Cancer Panel & 94 & 1700 exons and 284 SNPs & & - \\
\hline $\begin{array}{l}\text { Foundation } \\
\text { medicine }\end{array}$ & FoundationOne ${ }^{\circledR}$ & 315 & $\begin{array}{l}300 \text { cancer-related genes, including } \\
\text { select introns from more than } 25 \\
\text { genes often rearranged or altered } \\
\text { in solid tumors }\end{array}$ & 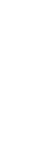 & Yes \\
\hline $\begin{array}{l}\text { Foundation } \\
\text { medicine }\end{array}$ & FoundationOne ${ }^{\circledR}$ Heme & 405 & Gene fusions & $\frac{1}{4}$ & - \\
\hline $\begin{array}{l}\text { Foundation } \\
\text { medicine }\end{array}$ & Foundation Focus ${ }^{\mathrm{TM}} \mathrm{CD} \times \mathrm{BRCA}$ & 2 & $B R C A 1$ and $B R C A 2$ mutations & $\frac{\bar{\varrho}}{\bar{n}}$ & Yes \\
\hline $\begin{array}{l}\text { Myriad } \\
\text { Genetics }\end{array}$ & BRCA Analysis & 2 & Gene mutations & $\frac{\grave{c}}{\frac{c}{c}}$ & Yes \\
\hline Illumina & $\begin{array}{l}\text { MiSeqDx Cystic Fibrosis Clinical } \\
\text { Sequencing Assay }\end{array}$ & 1 & $\begin{array}{l}\text { Intron/exon of the CFTR gene, } \\
\text { mutations, and INDELs }\end{array}$ & $\frac{ \pm 1}{3}$ & Yes \\
\hline Illumina & $\begin{array}{l}\text { Colorectal and Endometrial } \\
\text { Cancers }\end{array}$ & 19 & $\begin{array}{l}\text { Diagnostic/surveillance of Lynch } \\
\text { syndrome-like sporadic }\end{array}$ & $\frac{\bar{\sigma}}{\frac{0}{2}}$ & - \\
\hline Illumina & Extended RAS Panel & 2 & $\begin{array}{l}12 \text { codons in KRAS/NRAS, } 56 \\
\text { activating mutations }\end{array}$ & 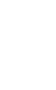 & Yes \\
\hline Ion Torrent & Ion Ampliseq hotspot & 46 & $\begin{array}{l}\text { Tumors suppressor genes and } \\
\text { oncogenes }\end{array}$ & $\frac{\digamma}{3}$ & - \\
\hline Illumina & TruSeq Amplicon & 48 & $\begin{array}{l}48 \text { genes are targeted with } 212 \\
\text { amplicons, oncogenes }\end{array}$ & $\frac{£}{\frac{\varrho}{2}}$ & - \\
\hline Ion Torrent & Various solid Tumor samples & 409 & $\begin{array}{l}\text { All exons of key tumors suppressor } \\
\text { genes and oncogenes }\end{array}$ & 党 & - \\
\hline & Immunology & & & ¿ & \\
\hline Illumina & $\begin{array}{l}\text { TruSight HLA Sequencing } \\
\text { Reproductive Health }\end{array}$ & HLA & 11 HLA loci & $\begin{array}{l}\frac{8}{0} \\
\frac{0}{3} \\
\frac{0}{2} \\
0 \\
\end{array}$ & - \\
\hline Illumina & $\begin{array}{l}\text { VeriSeq PGS } \\
\text { Genetic Health }\end{array}$ & & $\begin{array}{l}24 \text { chromosomes for selection of } \\
\text { embryos most likely to be euploid }\end{array}$ & $\begin{array}{l}\frac{1}{0} \\
\frac{0}{2} \\
\frac{0}{2} \\
\frac{0}{2}\end{array}$ & - \\
\hline Illumina & TruSight Inherited Disease & 552 & Mutation, intron-exon & 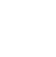 & - \\
\hline Illumina & TruSight One Sequencing & 4813 & $\begin{array}{l}\text { Coding regions of genes with } \\
\text { associated clinical phenotypes }\end{array}$ & 光 & - \\
\hline & Cardiomyopathy & & & $\frac{0}{3}$ & \\
\hline Illumina & TruSight Cardio Sequencing & 174 & $\begin{array}{l}\text { Cardiomyopathies, arrhythmias, } \\
\text { aortopathies, and more }\end{array}$ & $\frac{n}{\frac{n}{c}}$ & - \\
\hline
\end{tabular}

China Marrow Donor Program (The Data Bank of Chinese Hematopoietic Stem Cell Donors) for ultralarge-scale genotyping of donors indicating that NGS is cost-effective and a valuable tool ${ }^{10}$.

\section{BIOINFORMATICS}

A great challenge in NGS is bioinformatics analysis and data interpretation. Current NGS instruments can 
generate up to almost 1 terabase of information per run $\left(10^{12} \mathrm{bp}\right)$. These billions of sequences are then aligned and compared to a reference genome to identify variants or mutations ${ }^{11}$. These analyses require a solid bioinformatics infrastructure and personnel to handle the impressive amount of data generated for storage and analysis. One advantage of the commercial gene panels is that in many cases, they include access to online programs that perform the analysis and generate a clinical report. For example, SOPHiA DDM, a software platform from SOPHiA Genetics, detects, annotates, and pre-classifies raw genomic data to help clinicians to reach a better and faster diagnosis of patients across a broad range of diseases.

\section{NGS PLATFORMS AVAILABLE}

Among the several NGS systems currently available, including Pacific Biosciences, Oxford Nanopore, Ion Torrent (Thermo Fisher Scientific), and Illumina, the last two systems are the most employed in the clinical setting. The lon Torrent semiconductor-based sequencing technology has gained considerable acceptance and has recently launched the Global Ion Torrent Developers Alliance Program that provides the users with flexible options to access lon Torrent sequencing systems and the Ion AmpliSeq and Oncomine reagents. The lon Torrent Oncomine Dx Target Test is the first FDA-approved test that can detect multiple gene mutations in patients with metastatic non-small cell lung carcinoma. On the other hand, Illumina sequencing by synthesis chemistry has become the most widely used technology. The MiSeqDx System is the first FDA-cleared NGS platform for in vitro diagnostic testing, and a collection of panels for multiple diseases and the options for WGS or WES are available on all their platforms. In all cases, the instruments provide precise and reliable screening for diagnostic testing, with the speed and accuracy necessary for early disease detection, prognosis, or personalized treatment (Table 1). On a final note, the use of WES, WGS, or any type of sequencing has raised legal and ethical issues such as how and when to disclose incidental findings to the patient or study participant, and the data protection and access are still a concern.

\section{CONCLUSION}

The role of NGS in molecular diagnostics, disease prognosis, and pharmacologic therapy will increase further during the coming years. The advancement in sequencing technologies and the drop in sequencing costs will soon enable researchers to study genomes with greater coverage and depth and, more important, to follow an integrative genomics approach to associate genomic sequencing with transcriptomics and epigenomics data to identify clinically useful biomarkers for direct application in precision medicine.

\section{ACKNOWLEDGMENTS}

IAD-V is supported by a grant from PAPIIT: IA207017 and a fellowship by Fundación Salvador Zubirán. The authors thank Ari Kleinberg Bild from the Red de Apoyo a la Investigación, UNAM-INCMNSZ, Mexico, for his expert assistance in the preparation of figure 1.

\section{REFERENCES}

1. Rehm HL, Bale SJ, Bayrak-Toydemir P, et al. ACMG clinical laboratory standards for next-generation sequencing. Genet Med. 2013;15:733-47

2. Shen T, Pajaro-Van de Stadt SH, Yeat NC, Lin JC. Clinical applications of next generation sequencing in cancer: from panels, to exomes, to genomes. Front Genet. 2015;6:215.

3. Slavin TP, Niell-Swiller M, Solomon I, et al. Clinical application of multigene panels: challenges of next-generation counseling and cancer risk management. Front Oncol. 2015;5:208.

4. Hyman DM, Taylor BS, Baselga J. Implementing genome-driven oncology. Cell. 2017;168:584-99.

5. de Unamuno Bustos B, Murría Estal R, Pérez Simo G et al. Towards personalized medicine in melanoma: implementation of a clinical next-generation sequencing panel. Sci Rep. 2017;7:495.

6. Kumar-Sinha $C$, Chinnaiyan AM. Precision oncology in the age of integrative genomics. Nat Biotechnol. 2018;36:46-60.

7. Abou Tayoun AN, Spinner NB, Rehm HL, Green RC, Bianchi DW. Prenatal DNA sequencing: clinical, counseling, and diagnostic laboratory considerations. Prenat Diagn. 2018;38:26-32.

8. Domínguez-Vigil IG, Moreno-Martínez AK, Wang JY, Roehrl MH, Barrera-Saldaña HA. The dawn of the liquid biopsy in the fight against cancer. Oncotarget. 2017;9:2912-22.

9. Deurenberg RH, Bathoorn E, Chlebowicz MA, et al. Application of next generation sequencing in clinical microbiology and infection prevention. J Biotechnol. 2017;243:16-24.

10. Zhou M, Gao D, Chai X, et al. Application of high-throughput, high-resolution and cost-effective next generation sequencingbased large-scale HLA typing in donor registry. Tissue Antigens. 2015;85:20-8

11. Cummings CA, Peters E, Lacroix L, Andre F, Lackner MR. The role of next-generation sequencing in enabling personalized oncology therapy. Clin Transl Sci. 2016;9:283-92. 